\title{
A New Method for Determining the Reliability Testing Period Using Weibull Distribution
}

\section{Cristin Olimpiu Morariu, Sebastian Marian Zaharia}

Transilvania University of Brasov,

Faculty of Technological Engineering and Industrial Management, Department of Manufacturing Engineering

Colina Universitatii Nr. 1, 500036, Brasov, Romania

E-mail: zaharia_sebastian@unitbv.ro; c.morariu@unitbv.ro

\begin{abstract}
In this paper, we present a calculation methodology of the testing duration of the products' reliability, using the Weibull distribution, which allows the estimation of the mean duration of a censored and/or complete test, as well as of the confidence intervals for this duration. By using these values we can improve the adequate planning and allocation of material and human resources for the specific testing activities. The proposed methodology and the results' accuracy were verified using the Monte Carlo data simulation method.
\end{abstract}

Keywords: reliability; test plan; Weibull distribution; Monte Carlo simulation

\section{Introduction}

The reliability theory is a technological discipline closely related to the probability theory and mathematical statistics $[1,2,3]$. The data regarding the reliability of the products are obtained mainly through the following three methods: following the behavior of the products in real operation; during the laboratory tests; by using the data simulation through the Monte Carlo method. During the laboratory tests, we tried to emulate, as much as possible, the conditions in real operation, by reproducing the range of internal stresses, as well as the environmental stresses. The most important laboratory tests are the reliability tests $[4,5]$.

\subsection{Background on Reliability Test}

The reliability tests have a great importance, aiming either to determine, either to check the reliability characteristic of a product, if this is established in a predictive way. The reliability tests are extremely necessary and they have a decisive role in 
improving the technical solutions and in increasing the performances. The essential problem of reliability tests is the testing duration, which is generally comparable with the product's useful life time $[6,7,8,9]$.

The most used reliability tests are the following $[7,10,11,12,13]$ :

$>$ Complete tests (type $n$ out of $n$ ) - in these tests $n$ products of the same kind, the experiment being considered finished when all of the $n$ products have failed.

$>$ Censored tests (type $r$ out of $n$ ) - are commonly used and they consist of subjection to testing of $n$ products of the same type, the experiment being considered finished after the failure of $r<n$ tested products; obviously, the $r$ number is previously determined, usually by technical, economical and statistical considerations [2, 14, 15].

$>$ Truncated tests (with a fixed testing time) - a $n$ number of products are subjected to testing, but the experiment doesn't stop according to the number of failed elements, but according to a ${ }_{t r}$ time, previously set, a period during which the testing takes place.

The testing methodology about to be used has a direct economical impact, because in every test the following terms intervene: the cost of the tested product; the total cost of the experiment; the time consumed for testing and for the statistical processing of the data resulted from testing. Therefore, the selection of a specific type of test is a managerial decision that has to be taken by a responsible authority. Also, in the follow-up of the results of the statistical processing, we will propose certain corrective technical and economical actions, aimed directly at the quality and reliability of the product in question $[3,6,16,17,18,19,20]$.

In order to realize reliability tests we must take into account the following aspects:

a. A previously determined $n$ number of products that will be subjected to testing.

b. A testing plan that includes the following aspects: the selection of stress parameters, which will determine the failure mechanisms specific to the product.

c. Instructions regarding the adequate type of test and the methods of calculation, in order to estimate the reliability parameters.

d. A test chart where the experimental data are recorded and the statistical calculations, as well as the chronological recording of observations and interventions, are made.

e. Testing stands, testing equipment, auxiliary materials and qualified staff in order to realize the test. 


\subsection{Nomenclature}

$W(t, \beta, \eta)$ - the Weibull distribution, having the function of distribution:

$$
F(t)=1-e^{-\left(\frac{t}{\eta}\right)^{\beta}} ;
$$

$\beta$ - the shape parameter of Weibull distribution;

$\eta \quad$ - the scale parameter of Weibull distribution;

$B(x, n, p) \quad$ - the binomial distribution, having the function of probability:

$$
\operatorname{Pr}(X=x)=C_{n}^{x} \cdot p^{x} \cdot(1-p)^{n-x}
$$

$N$ - the number of simulations;

$n$ - the sample volume;

$r$ - the level of censorship;

$F_{n}\left(t_{i}\right)$ - the empirical function of distribution correspondent to the operation time until failure;

$T_{n / n}$ - the duration of a complete test realized on a volume sample $n$;

$T_{r / n}$ - the duration of a censored test al level $r$, realized on volume $n$ sample;

$1-\alpha \quad$ - confidence level;

$\alpha \quad$ - significance level;

$Q_{p, v l, v 2}$ - the p quantile of the Fisher - Snedecor, with $\mathrm{v}_{1}$ and $\mathrm{v}_{2}$ degrees of freedom;

$U=1-\alpha / 2 \quad$ - the index used to note the superior confidence limit; represents the value of the probability corresponding to the estimation of the superior limit of the testing duration, [\%];

$L=\alpha / 2 \quad$ - the index used to note the inferior confidence limits; represents the value of the probability corresponding to the estimation of the inferior limit of the testing duration, [\%].

\subsection{Review on the Calculus of the Testing Period}

When using the Weibull distribution in the modeling of the products' reliability, these is estimated, in the majority of situations, on the basis of experimental results obtained following their testing on stands, using censored tests or complete tests. The organization and the process of reliability tests of the products represent 
complex activities from an organizational standpoint and also big resources consumers. The calculation relations of the duration of a complete or a censored reliability test, found in the specialty literature, are based on a series of equations which allow the estimation of the empirical distribution function [9]:

$$
F_{n}\left(t_{r}\right)=\frac{r}{n+1}
$$

The equation (1) gives mean values of the empirical distribution function. The mean values of the empirical distribution function can be obtained using:

$$
F_{n}\left(t_{r}\right)=\frac{r-0.3}{n+0.4}
$$

or:

$$
F_{n}\left(t_{r}\right)=\frac{i-0.30685-0.3863 \cdot\left(\frac{r-1}{n-1}\right)}{n},
$$

for $n>20$ and:

$$
F_{n}\left(t_{r}\right)=1-2^{-\frac{1}{n}}\left(\frac{r-1}{n-1}\right) \cdot\left[2^{\left(1-\frac{1}{n}\right)}-1\right]
$$

for $n \leq 20$.

The value of the duration of a reliability test censored at level $r$ is obtained using the inverse function of distribution of the considered statistical model [4, 21, 22]:

$$
\mathrm{T}_{r / n}=\eta \cdot\left\{\ln \left[\frac{1}{1-F_{n}\left(t_{r}\right)}\right]\right\}^{\frac{1}{\beta}},
$$

in this case being the Weibull distribution. The equation (5) results from the logarithmation of the Weibull distribution function, written as follows:

$$
e^{-\left(\frac{t}{\eta}\right)^{\beta}}=1-F(t)
$$

we obtain:

$$
\left(\frac{t}{\eta}\right)^{\beta}=\ln \left[\frac{1}{1-F(t)}\right] \text {. }
$$


We notice that after a series of algebraic calculations, the equation (7) can be written in the form of equation (5). Also, in the equation (5), instead of $F(t)$ we used the value determined by using one of the $(1) \div(4)$ relations. Thus we obtained mean or median values of the duration of testing. For complete test case in relations (1) $\div(4)$, parameter $r$ is replaced by the value of the sample volume used $n$. Consequently, the objective of this paper is to present an estimation modality of the mean duration for censored and/or complete reliability tests, as well as of the confidence intervals for this duration. Knowing these values allows for the careful planning of the testing activities [4, 23, 24].

\section{Statistical Calculation Model}

The value obtained for a reliability test doesn't offer important data regarding the real duration of a test, because the time of operation until failure of a tested product represents a random variable.

For this situation, a favorable solution consists is the determination of the confidence intervals of the duration of the test. These intervals contain the real value of the test, with a $1-\alpha$ probability $[25,26]$ :

$$
\operatorname{Pr}\left(\mathrm{T}_{L} \leq \mathrm{T}_{r / n} \leq \mathrm{T}_{U}\right)=1-\alpha .
$$

The calculation of the confidence intervals is realized in the conditions of a Bernoulli extraction (the scheme of the urn with returned balls). Thus, the median value of probability at which from $n$ products subjected to testing, a number of $r$ products fail, results as a solution to the equation [4, 27, 28]:

$$
\sum_{i=r}^{n} C_{n}^{i} \cdot F_{M e}^{i} \cdot\left(1-F_{M e}\right)^{n-i}=0.5 .
$$

The difficulties in calculation which can occur solving the equation (9), depending on $F_{M e}$, can be eliminated by using an approximate value:

$$
F_{M e}=\frac{1}{1+\frac{n-r+1}{r} \cdot Q_{0.5,2 \cdot(n-r+1), 2 \cdot r}} .
$$

The equation (10) represents the connecting relation that can be established between the binomial distribution and the Fisher-Snedecor distribution [4, 11, 26].

Using the $F_{M e}$ solution, obtained by solving one of the (9) or (10) equations, along with to equation (5), leads to the obtaining of the duration of the reliability test. In fact, the equations (1) $\div$ (4) are nothing more than regression relations of the solutions of equation (9), for different combinations of the parameters $n$ and $r$. 
Because, by definition, the distribution function is an ascending function, the confidence level of the duration of testing period results by using the solutions of the equations:

$\sum_{i=r}^{n} C_{n}^{i} \cdot F_{L}^{i} \cdot\left(1-F_{L}\right)^{n-i}=\frac{\alpha}{2}$,

and

$$
\sum_{i=r}^{n} C_{n}^{i} \cdot F_{U}^{i} \cdot\left(1-F_{U}\right)^{n-i}=1-\frac{\alpha}{2},
$$

together with (5), namely:

$$
\mathrm{T}_{L}=\eta \cdot\left\{\ln \left[\frac{1}{1-F_{L}}\right]\right\}^{\frac{1}{\beta}},
$$

and

$$
\mathrm{T}_{U}=\eta \cdot\left\{\ln \left[\frac{1}{1-F_{U}}\right]\right\}^{\frac{1}{\beta}},
$$

A similar value of the $F_{L}$ and $F_{U}$ probabilities can be obtained by approximating the binomial distribution through the Fisher-Snedecor distribution:

$$
F_{L}=\frac{1}{1+\frac{n-r+1}{r} \cdot Q_{1-\frac{\alpha}{2}, 2 \cdot(n-r+1), 2 \cdot r}} .
$$

and

$$
F_{U}=\frac{1}{1+\frac{n-r+1}{r} \cdot Q_{\frac{\alpha}{2}, 2 \cdot(n-r+1), 2 \cdot r}} .
$$

For the case of the complete tests, in the calculation relations (10) and (15), (16) the parameter $r$ is replaced with the value of the used sample volume $n$. 


\section{Simulation Study}

\subsection{Program Description}

To verify the precision of reasoning and of the mathematic model proposed at point 2, we used the Monte Carlo simulation method. The method implies, in this case, generating a very high number of samples $(\mathrm{N}>>1000)$, that belong to a completely specified Weibull population, $\mathrm{W}(t, \beta, \eta)$. This database is then subjected to a statistical analysis that is aimed at the duration of a reliability test using a censored plan with the $n$ and $r$ parameters. For the development of this study we created a Mathcad calculus programme. The logical chart of this programme is presented in Figure 1. The running of the programme implies the determination of the following entry data: $N, n, r, \beta, \eta$ and $\alpha$. The program generates a matrix with $\mathrm{n}$ lines and $\mathrm{N}$ columns, using the generator of random, uniform and continuous numbers within the $[0,1]$ interval. The values of simulated failure times are obtained by using the inverse function of distribution of the Weibull statistical:

$t=\eta \cdot \ln \left(\frac{1}{1-r n d(1)}\right)^{\frac{1}{\beta}}$.

Thus, we obtain a matrix with $n x N$ dimensions, in which every column represents a reliability test. In order to determine the duration of censored tests at level $r$, the calculation program sets in ascending order the columns of the previously generated matrix.

Also, the $r$-th line of this matrix is extracted at the end. The $N$ values contained in this line represent the simulated durations of the reliability tests $\left(t_{r}, i\right)$. The calculation of the median and mean durations of the testing duration is made by determining the median and the mean of these values:

$t_{M e}= \begin{cases}t_{\left(\frac{n+1}{2}\right)}, & \text { if } N \text { is even } \\ \frac{t_{\left(\frac{n}{2}\right)}+t_{\left(\frac{n}{2}+1\right)},}{2}, & \text { if } N \text { is odd }\end{cases}$

and

$m=\frac{\sum_{i=1}^{N} t_{r, i}}{N}$ 


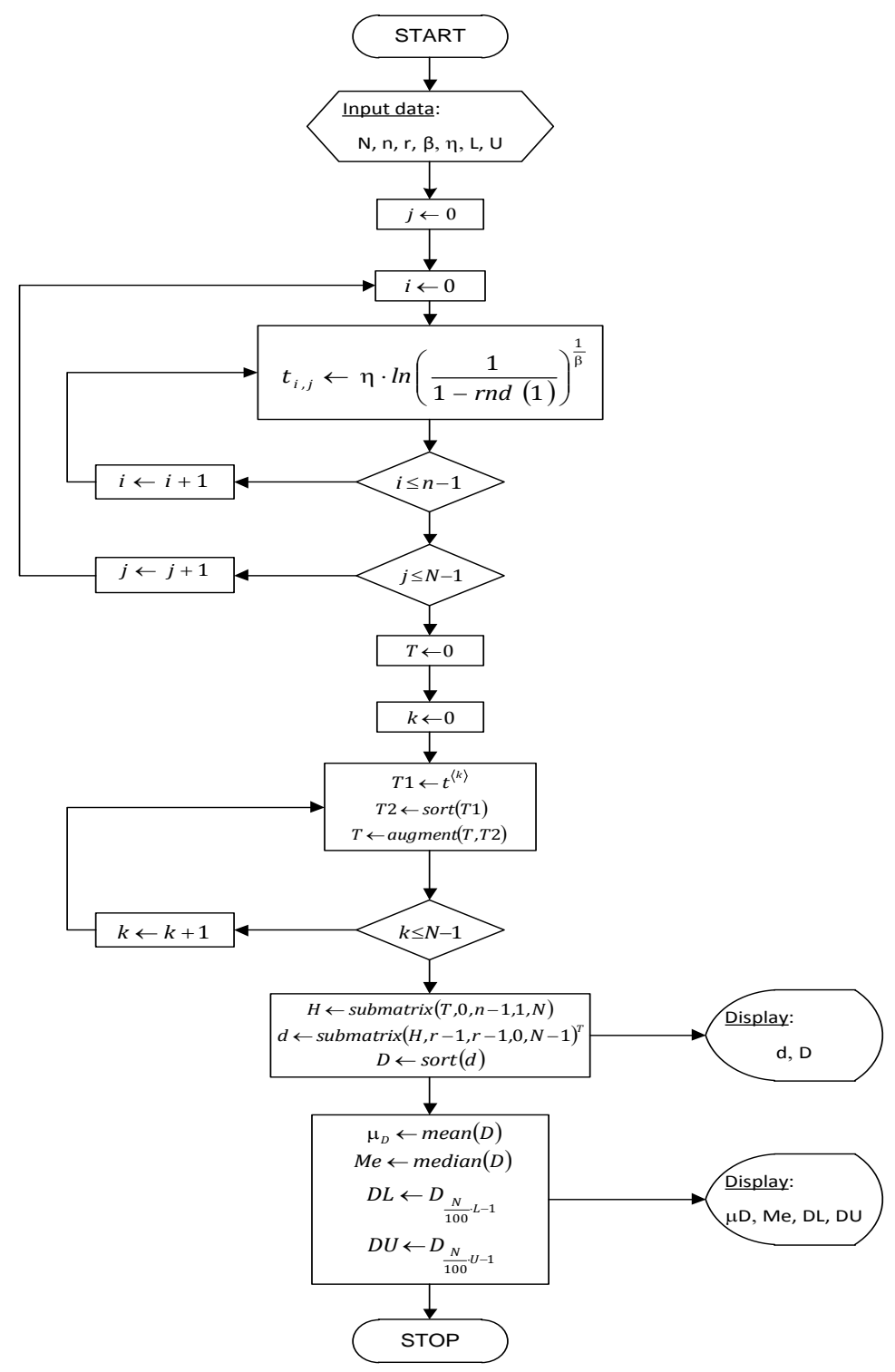

Figure 1

The logical scheme of the Monte Carlo numerical simulation program

In the previous equations, we noted $t_{(p)}$ as the $p$ quantile of the $t$ variable. The determination of confidence limits for the duration of the tests is realized by determining the $t_{L / 100}$ and $t_{U / 100}$ quantiles of the truncation durations for the $N$ simulated tests. The calculation method used for the determination of $p$ quantiles applies the equation: 
$t_{p}=t_{(p \cdot(N+1))}$.

If the $p \cdot(n+1)$ expression doesn't generate an integer value, then for the determination of the $p$ quantile we recommend the use of linear interpolation. We assume that, after the evaluation of the $p \cdot(n+1)$ expression, we find that the value of the $t_{p}$ quantile is included in the $\left[t_{(k),} t_{(k+1)}\right]$. To determine the value of the $t_{p}$ quantile, we use the relation:

$t_{p}=t_{(k)}+[p \cdot(n+1)-k] \cdot\left[t_{(k+1)}-t_{(k)}\right]$

For high volumes of sample (such is the case with the realized application), instead of the previous relation we can use equation (22).

$t_{p}=t_{[p \cdot(N+1)]} \cdot$

In equation (22), by $t_{[\mathrm{p}]}$ we noted the integer part of the value of the expression between brackets.

\subsection{Monte Carlo Simulation Data}

To demonstrate the way of using the calculation methodology presented in the third point of this paper, we present further several case studies, determined for different values of the Weibull distribution parameters $\beta$ and $\eta$, as well as for different testing schemes $n$ and $r$.

The solving of the equations (9), (11) and (12) was made using the specialized functions existent in Mathcad 14. The solving accuracy of these equations was established at $10^{-15}$. In parallel, we presented the values obtained by using the approximate relations (10), (15) and (16).

The obtained $F_{M e}, F_{L}$ şi $F_{U}$ probabilities are then used to determine the median duration of the reliability test, eq. (5) and the limits of the confidence interval (1$\alpha$ ) for this duration $\left(T_{L}\right.$ şi $\left.T_{U}\right)$.

The values for these limits are obtained by using the equations (13) and (14). The significance level was established at the value of $\alpha=10 \%$. In Table 1 values for different combinations of the Weibull distribution's parameters and different censored testing plans, $n / r$ are presented.

The accuracy of the obtained results, by using the proposed calculation methodology, was verified using the Monte Carlo simulation.

For this purpose we used the MathCAD 14 software, which is described at point 3.1. The calculus programme was run for the same combinations of values of the Weibull distribution's parameters, as in the previous case. Also, the number of simulations was established at the value $\mathrm{N}=10000$ and the confidence interval $1-\alpha$ at $90 \%$. 
Table 1

The determination of the testing durations related with the reliability tests using the calculation relations

\begin{tabular}{|c|c|c|c|c|c|c|c|}
\hline \multirow{2}{*}{\multicolumn{2}{|c|}{ Test plan }} & \multicolumn{6}{|c|}{ The calculated duration of the reliability test } \\
\hline & & \multicolumn{2}{|c|}{$T_{r / n}$} & \multicolumn{2}{|c|}{$T_{L}$} & \multicolumn{2}{|c|}{$T_{U}$} \\
\hline$n$ & $r$ & eq. (8) & eq. (9) & eq. (10) & eq. (14) & eq. (11) & eq. (15) \\
\hline \multicolumn{8}{|c|}{$\beta=1.5, \eta=50$} \\
\hline 10 & 5 & 35.605 & 35.605 & 19.926 & 19.926 & 56.218 & 56.218 \\
\hline 10 & 10 & 97.035 & 97.035 & 61.118 & 61.118 & 151.520 & 151.520 \\
\hline 20 & 5 & 20.417 & 20.417 & 11.472 & 11.472 & 32.023 & 32.023 \\
\hline 20 & 10 & 37.332 & 37.332 & 25.278 & 25.278 & 51.936 & 51.936 \\
\hline 20 & 15 & 58.860 & 58.860 & 42.591 & 42.591 & 78.556 & 78.556 \\
\hline 20 & 20 & 112.601 & 112.601 & 78.641 & 78.641 & 164.494 & 164.494 \\
\hline \multicolumn{8}{|c|}{$\beta=1.5, \eta=100$} \\
\hline 10 & 5 & 71.211 & 71.211 & 39.854 & 39.854 & 112.438 & 112.438 \\
\hline 10 & 10 & 194.069 & 194.069 & 122.236 & 122.236 & 303.040 & 303.040 \\
\hline 20 & 5 & 40.834 & 40.834 & 22.945 & 22.945 & 64.045 & 64.045 \\
\hline 20 & 10 & 74.664 & 74.664 & 50.556 & 50.556 & 103.871 & 103.871 \\
\hline 20 & 15 & 117.720 & 117.720 & 85.182 & 85.182 & 157.112 & 157.112 \\
\hline 20 & 20 & 225.201 & 225.201 & 157.282 & 157.282 & 328.989 & 328.989 \\
\hline \multicolumn{8}{|c|}{$\beta=2, \eta=50$} \\
\hline 10 & 5 & 38.760 & 38.760 & 25.080 & 25.080 & 54.595 & 54.595 \\
\hline 10 & 10 & 82.213 & 82.213 & 58.126 & 58.126 & 114.841 & 114.841 \\
\hline 20 & 5 & 25.541 & 25.541 & 16.576 & 16.576 & 35.796 & 35.796 \\
\hline 20 & 10 & 40.161 & 40.161 & 29.978 & 29.978 & 51.445 & 51.445 \\
\hline 20 & 15 & 56.508 & 56.508 & 44.333 & 44.333 & 70.166 & 70.166 \\
\hline 20 & 20 & 91.917 & 91.917 & 70.223 & 70.223 & 122.139 & 122.139 \\
\hline \multicolumn{8}{|c|}{$\beta=2, \eta=100$} \\
\hline 10 & 5 & 91.917 & 91.917 & 70.223 & 70.223 & 122.139 & 122.139 \\
\hline 10 & 10 & 164.425 & 164.425 & 116.252 & 116.252 & 229.681 & 229.681 \\
\hline 20 & 5 & 51.082 & 51.082 & 33.152 & 33.152 & 71.592 & 71.592 \\
\hline 20 & 10 & 80.322 & 80.322 & 59.956 & 59.956 & 102.890 & 102.890 \\
\hline 20 & 15 & 113.015 & 113.015 & 88.667 & 88.667 & 140.332 & 140.332 \\
\hline 20 & 20 & 183.835 & 183.835 & 140.446 & 140.446 & 244.279 & 244.279 \\
\hline
\end{tabular}

Under these conditions, we determined the median values, the mean values and the confidence intervals for the testing duration. The results obtained by Monte Carlo numerical simulation are presented in Table 2. 
Table 2

The determination of the testing durations related to the reliability tests using the Monte Carlo method

\begin{tabular}{|c|c|c|c|c|c|}
\hline \multicolumn{2}{|c|}{ Test plan } & \multicolumn{4}{|c|}{ Values obtained by Monte Carlo simulation } \\
\hline & & $T_{r / n}$ & $T_{L}$ & $T_{U}$ & $\check{T}_{r / n}$ \\
\hline$n$ & $r$ & & & & \\
\hline \multicolumn{6}{|c|}{$\beta=1.5, \eta=50$} \\
\hline 10 & 5 & 35.744 & 19.998 & 55.945 & 36.562 \\
\hline 10 & 10 & 96.820 & 61.412 & 152.861 & 100.609 \\
\hline 20 & 5 & 20.385 & 11.680 & 31.748 & 20.885 \\
\hline 20 & 10 & 37.301 & 25.362 & 52.071 & 37.843 \\
\hline 20 & 15 & 58.992 & 42.416 & 78.349 & 59.594 \\
\hline 20 & 20 & 112.252 & 78.947 & 165.037 & 115.808 \\
\hline \multicolumn{6}{|c|}{$\beta=1.5, \eta=100$} \\
\hline 10 & 5 & 71.140 & 39.118 & 111.946 & 72.881 \\
\hline 10 & 10 & 193.087 & 121.367 & 301.877 & 200.325 \\
\hline 20 & 5 & 40.782 & 22.834 & 63.778 & 41.770 \\
\hline 20 & 10 & 74.810 & 50.408 & 103.875 & 75.681 \\
\hline 20 & 15 & 118.342 & 85.409 & 158.009 & 119.295 \\
\hline 20 & 20 & 225.390 & 157.378 & 328.730 & 231.811 \\
\hline \multicolumn{6}{|c|}{$\beta=2, \eta=50$} \\
\hline 10 & 5 & 38.614 & 25.034 & 54.654 & 39.101 \\
\hline 10 & 10 & 82.338 & 58.557 & 115.534 & 83.971 \\
\hline 20 & 5 & 25.502 & 16.507 & 35.797 & 25.793 \\
\hline 20 & 10 & 40.211 & 29.926 & 51.526 & 40.393 \\
\hline 20 & 15 & 56.463 & 44.296 & 70.287 & 56.759 \\
\hline 20 & 20 & 91.957 & 70.221 & 121.616 & 93.419 \\
\hline \multicolumn{6}{|c|}{$\beta=2, \eta=100$} \\
\hline 10 & 5 & 91.957 & 70.221 & 121.616 & 93.419 \\
\hline 10 & 10 & 164.752 & 116.708 & 228.715 & 167.494 \\
\hline 20 & 5 & 51.307 & 33.260 & 71.201 & 51.696 \\
\hline 20 & 10 & 80.452 & 59.513 & 102.790 & 80.616 \\
\hline 20 & 15 & 113.174 & 88.908 & 139.999 & 113.716 \\
\hline 20 & 20 & 183.619 & 140.521 & 244.166 & 186.904 \\
\hline
\end{tabular}

The using mode of this method for the estimation of the durations of the complete and/or at $\mathrm{r}$ level censored reliability tests is presented in Figure 2. 
1. The determination of the a priori values of the Weibull distribution parameters: $\beta, \eta$

distribution parameters: $\beta, \eta$
2. The determination of the parameters of the realized test: $n, r$
3. The determination of the confidence level: $1-\alpha$
4. The calculation of the median probability of the testing time, eq.
(10) and of the median value of the testing duration, eq. (5)
5. The calculation of the $\mathrm{F}_{L}$ probability, eq. (15) and of the inferior
limit if the testing duration, eq. (13)
6. The calculation of the $\mathrm{F}_{U}$ probability, eq. (16) and of the
superior limit of the testing duration, eq. (14)

Figure 2

The calculation algorithm for the duration of the reliability tests

\section{Conclusions}

Based on the results presented in Tables 1 and 2, we will realize several comparative studies to show the correctness of the proposed calculus method. In Figure 3 the results of the median duration of a censored test at level $r$ are presented, realized on a sample of $n$ volume, on a graphical form for the case study $\beta=1.5, \eta=50$. The inferior and superior limits of the testing duration for the case study in question $(\beta=1.5, \eta=50)$ are presented in Figures 4 and 5.

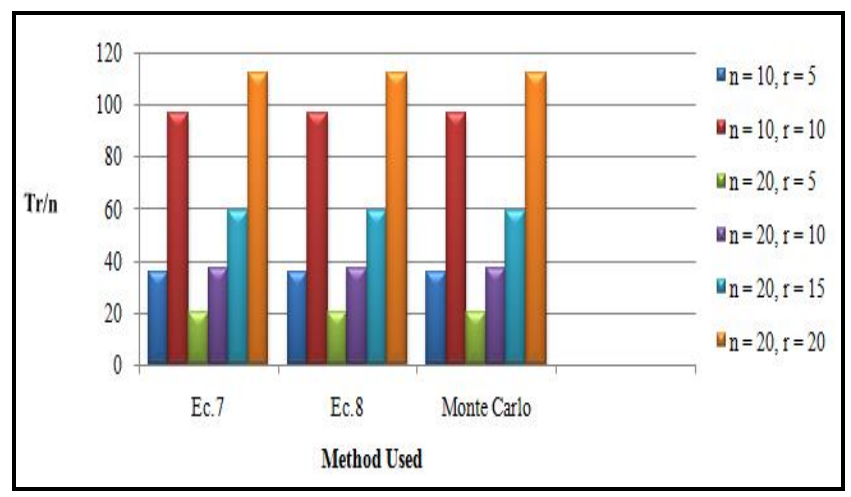

Figure 3

The duration of a censored test level $r$, realized on sample size $n\left(T_{n / r}\right)$ 


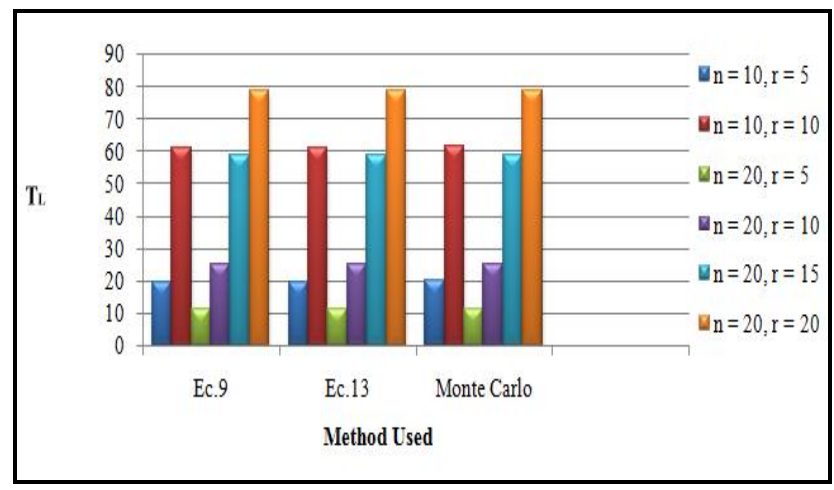

Figure 4

Limit inferior duration of a censored test level $\mathrm{r}$, realized on sample size $n\left(T_{L}\right)$

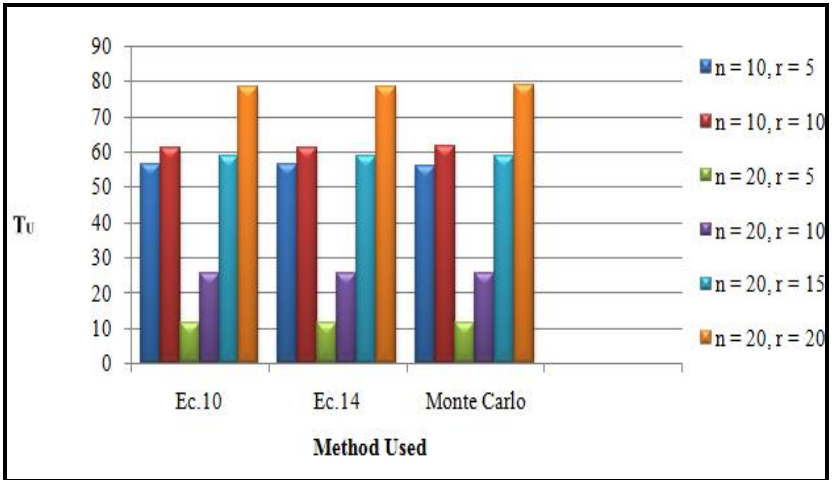

Figure 5

Limit superior duration of a censored test level r, realized on sample size $n\left(T_{U}\right)$

The presented calculation model allows the obtaining of accurate estimated values, because the differences towards the simulated values are very small. If the number of simulations would grow, the resulting differences would be insignificant. The approximate relations (10), (15) and (16) lead to the obtaining of some values, which, at the results' display accuracy of $10^{-3}$, don't differ from the real values obtained through the equations (9), (11), (12). Based on the presented results we found the significant reductions in time that can be made by using the censored testing plans.

Given the powerful competition on the industrial market, we can no longer imagine the realization of a product without a rigorous quality and reliability control of the product, based on different types of tests, in all the stages of the products' existence, from the raw materials being used, up to their use. In these types of tests, we put special emphasis on the reliability tests. The testing 
laboratories, that possess modern testing equipment and highly-trained personnel, have acquired an ever increasing development. Today, almost every company has a reliability test laboratory, adequately equipped to the type of products it realizes. The application of censored testing plans, using the Monte Carlo method, determines the testing duration of the products in a shorter time and in conditions of economic efficiency.

The proposed method of calculation has applicability in laboratories that are specialized in testing the materials or the products from different fields of use. These fields are given by the particular versatility of the Weibull distribution's model:

* the tear resistance, the corrosion resistance, the wear resistance, the fatigue resistance and the contact fatigue resistance of textile and metallic materials;

* the modeling of the materials properties: steels, titanium, semi-conductor materials, tungsten, ceramic, glass, plastic materials, porcelain, graphite, paper, textile fibers, composite materials;

* the modeling of the durability of mechanical components: bearings, engines, motor vehicles' structures, tools;

* the modeling of the functioning times of relays, passive electronic components (resistors and capacitors) and active electronic components (transistors, integrated circuits);

* the modeling of the life times of subsystems, made of identical component elements, in series connected and whose behaviour is described using the gamma distribution.

\section{Acknowledgement}

This paper is supported by the Sectoral Operational Programme Human Resources Development (SOP HRD), financed from the European Social Fund and by the Romanian Government under the project number POSDRU/89/1.5/S/59323.

\section{References}

[1] D. W. Benbow. The Certified Reliability Engineer Handbook, ASQ Quality Press, Milwaukee, 2009

[2] B. Bertsche. Reliability in Automotive and Mechanical Engineering: Determination of Component and System Reliability, Springer, Berlin, 2010

[3] P. O’Connor \& A. Kleyner. Practical Reliability Engineering, $5^{\text {th }}$ Edition. Wiley \& Sons, New Jersey, 2012

[4] D. B. Kececioglu. Reliability Engineering Handbook, Vol. I, PTR PrenticeHall, New Jersey, 1991 
[5] G. B. Yang. Life Cycle Reliability Engineering. New Jersey, Wiley, 2007

[6] I. Arizonoa, Y. Kawamuraa, Y. Takemotob. Reliability Tests for Weibull Distribution with Variational shape Parameter Based on Sudden Death Lifetime Data, European Journal of Operational Research, 189(2): 570574, 2008

[7] E. E. Lewi. Introduction to Reliability Engineering, Wiley, New Jersey, 1995

[8] S. C. Saunders. Reliability, Life Testing and the Prediction of Service Lives, Springer, New York, 2007

[9] E. Zio. An Introduction to the Basics of Reliability and Risk Analysis, World Scientific, New Jersey, 2007

[10] A. Joarder, H. Krishna, D. Kundu. Inferences on Weibull Parameters with Conventional Type-I Censoring, Computational Statistics \& Data Analysis, 55(1): 1-11, 2011

[11] D. B. Kececioglu. Reliability \& Life Testing Handbook, Vol. II, PTR Prentice-Hall, New Jersey, 1994

[12] C. Kim, J. Jung, Y. Chung. Bayesian Estimation for the Exponentiated Weibull Model Under Type II Progressive Censoring, Statistical papers, 52(1): 53-70, 2011

[13] S. M. K. Quadri \& N. Ahmad. Software Reliability Growth Modeling with New Modified Weibull Testing-effort and Optimal Release Policy, International Journal of Computer Applications, 6(12):1-10, 2010

[14] M. I. Ageel. A Novel Means of Estimating Quantiles for 2 - Parameter Weibull Distribution under the Right Random Censoring Model, Journal of Computational and Applied Mathematics, 149(2): 373-380, 2002

[15] D. Kundu. Bayesian Inference and Life Testing Plan for the Weibull Distribution in Presence of Progressive Censoring, Technometrics, 50(2): 144-154, 2008

[16] H. Chin-Yu. Cost-Reliability-Optimal Release Policy for Software Reliability Models Incorporating Improvements in Testing Efficiency, The Journal of Systems and Software, 77 (2):139-155, 2005

[17] P. C. Jha, D. Gupta, B. Yang, P. K. Kapur. Optimal Testing Resource Allocation during Module Testing Considering Cost, Testing Effort and Reliability, Computers \& Industrial Engineering, 57(3): 1122-1130, 2009

[18] A. Kleynera \& P. Sandborn. Minimizing Life Cycle Cost by Managing Product Reliability via Validation Plan and Warranty Return Cost, Int. J. Production Economics, 112(2): 796-807, 2008 
[19] Y. I. Kwon. A Bayesian Life Test Sampling Plan for Products with Weibull Lifetime Distribution Sold under Warranty, Reliability Engineering \& System Safety, 53(1): 61-66, 1996

[20] S. M. Zaharia, I.Martinescu, C. O. Morariu. Life Time Prediction using Accelerated Test Data of the Specimens from Mechanical Element. Eksploatacja i Niezawodnosc - Maintenance and Reliability; 14(2): 99-106, 2012

[21] H. S. Alkutubi \& H. M. Ali. Maximum Likelihood Estimators with Complete and Censored Data, European Journal of Scientific Research, 54(3): 407-410, 2011

[22] H. Panahi \& S. Asadi. Estimation of the Weibull Distribution Based on Type-II Censored Samples, Applied Mathematical Sciences, 5(52): 25492558, 2011

[23] O. P. Yadav, N. Singh, P. S. Goel. Reliability Demonstration Test Planning: A Three Dimensional Consideration, Reliability Engineering and System Safety, 91(8): 882-893, 2006

[24] G. Yang \& L. Jin. Best Compromise Test Plans for Weibull Distributions with Different Censoring Times, Quality and Reliability Engineering International, 10(5): 411-415, 1994

[25] C. O. Morariu \& T. Păunescu. Computer Applications in Engineering Mathcad 2001, Ed. Transilvania University of Braşov, 2004

[26] C. O. Morariu. Applied Probability and Statistics, Ed. Transilvania University of Braşov, 2010

[27] G. C. Perdona \& F. Louzada - Neto. Interval Estimation for the Parameters of the Modified Weibull Distribution Model with Censored Data: a Simulation Study, TEMA Tend. Mat. Apl. Comput, 9(3): 437-446, 2008

[28] S. Vittal \& R. Phillips. Uncertainty Analysis of Weibull Estimators for Interval-Censored Data, Reliability and Maintainability Symposium RAMS'07, pp. 292-297, 22-25, Jan. 2007 